DO-TH 00/12

hep-ph/0008044

\title{
Cancellations in Neutrinoless Double Beta Decay and the Neutrino Mass Matrix
}

\author{
Werner Rodejohann* \\ Lehrstuhl für Theoretische Physik III, \\ Universität Dortmund, Otto-Hahn Str. 4, \\ 44221 Dortmund, Germany
}

\begin{abstract}
In a degenerate scheme with mass $m_{0}$ a complete analysis of the allowed range of the effective electron neutrino Majorana mass $\langle m\rangle$ is performed. Special attention is paid to effects of cancellations caused either by intrinsic $C P$ parities of the eigenstates ( $C P$ invariance) or by complex mixing matrix elements ( $C P$ violation). We investigate all possibilities and give in each case constraints on the phases, the relative $C P$ parities or the neutrino mass scale. A solar mixing angle $\sin ^{2} 2 \theta$ smaller than 0.7 jeopardizes the degenerate mass scheme. A key value of $\langle m\rangle / m_{0}$ is identified, which is independent on the solar solution and would rule out certain schemes. Also it would answer the question regarding the presence of $C P$ violation. Even if a total neutrino mass scale and an effective mass is measured, the value of the phases or parities is not fixed, unless in some special cases. The resulting uncertainty in the other mass matrix elements is at least of the same order than the one stemming from nuclear matrix elements calculations.
\end{abstract}

Keywords: Neutrino Oscillation, Double Beta Decay, Massive Neutrinos, Majorana Neutrinos PACS: 14.60.Pq, 23.40.-s

\footnotetext{
*Email address: rodejoha@xena.physik.uni-dortmund.de
} 


\section{Introduction}

In the light of recent impressive experimental evidence on neutrino oscillations [1] the next fundamental question to be answered is the one regarding the neutrino character. From the theoretical side Majorana neutrinos are favored, since they pop out of almost every GUT and are for example the result of the very attractive see-saw-mechanism [2]. In this case, heavy neutrinos are predicted. Experimental information on the existence of Majorana neutrinos might come from neutrinoless double beta decay $(0 \nu \beta \beta)$ [3] or from production of heavy Majorana neutrinos at colliders, see e.g. [4] and references therein. The most stringent limit however comes from the first process, investigating the effective Majorana mass of the electron neutrino, with a current bound of [5]

$$
\langle m\rangle \lesssim 0.2 \mathrm{eV}
$$

Plans exist to build experiments exploring regions up to $\langle m\rangle \simeq 0.002 \mathrm{eV}$ [6]. The variation within a factor of roughly 3 between different calculations of the required nuclear matrix elements has to be kept in mind. Results of oscillation experiments can be used to restrict the value of $\langle m\rangle$ in different mass schemes and for the different solutions of the solar neutrino problem [7, 8, 9, 10, 11, 12, 13, 14, 15, 16, 17]. Typical key scales for $\langle m\rangle$ are 0.1 and $0.005 \mathrm{eV}$, thus lying in the range of current and forthcoming projects. However, $\langle m\rangle$ has a form which includes possibilities for the contributions to cancel each other, namely via $C P$-violating phases or via the intrinsic $C P$-parities of the mass states, which exist in the case of $C P$ conservation. These effects were included in most of the above given references to get the maximal and minimal values of $\langle m\rangle$. In [15] constraints on $C P$-violating phases were given by using a graphical representation for the complex mass matrix elements, although without applying numerical values. Numerical studies regarding the Majorana phases were given in [8, 10]. Some overlap with these works exists, however, we give many plots and statements, which are new to the literature and adopt an approach, which allows to investigate different situations regarding measurements of $\langle m\rangle$ and/or $m_{0}$. Here $m_{0}$ denotes the common mass scale in a degenerate mass scheme. Among the topics discussed are a clarification of the kind of cancellation, i.e. a distinction between the 2 possibilities. When starting from a degenerate scheme and in a given situation the maximal allowed $m_{0}$ is comparable to the scale implied by the atmospheric anomaly, so the degenerate scheme is ruled out and the derived mass limit holds for the largest of the 3 mass eigenstates. We will find that only in very special cases definite statements about the phases or parities can be made and that alone from this fact there is a large uncertainty in the values of the other mass matrix entries. This uncertainty is ranging from 2 to factors around 20. The other entries are clearly needed to distinguish between different discussed models. The only information about the phases or parities can come from neutrinoless double beta decay: All other mass matrix entries are impossible to measure directly, since the respective branching ratios or cross sections they govern are way beyond experimental access [14]. Apart from the obvious aspect that fundamental parameters of a model need to be measured, other

interesting application of the phases exist: In [18] it was found that the Majorana phases play a crucial role in the stability of the mixing angles against quantum corrections. Ref. 
[19] finds that their values have influence on the magnitude of the lepton-asymmetry in the universe, which can be made responsible for the observed baryon-asymmetry. Thus, the precise knowledge of all mixing parameters in the lepton sector is certainly important. The paper is organized as follows: In Section 2 we present the general framework and basic formulae for $C P$ violation and conservation, respectively. Section 3 sees a discussion of the connection between oscillation and $\langle m\rangle$ in hierarchical schemes, whereas Sec. $\mathbb{E}$ concentrates on the degenerate scheme. Some special mixing matrices and the general case with experimentally favored values are discussed. For each case constraints on the phases, parities and $m_{0}$ are given. The paper is concluded in Sec. 5 with a summary of our results.

\section{Formalism}

Flavor eigenstates $\nu_{\alpha}(\alpha=e, \mu, \tau)$ are connected to mass eigenstates $\nu_{i}(i=1,2,3)$ via a mixing matrix, i.e. $\nu_{\alpha}=S_{\alpha i} \nu_{i}$. A proper treatment of this issue can be found in [20, here we quote only the main points. This matrix is derived by diagonalising the Majorana mass term in the Langrangian:

$$
-\mathcal{L}_{\text {mass }}=\frac{1}{2} M_{\alpha \beta} \overline{\nu_{\alpha L}} \nu_{\beta R}^{c}+\text { h.c. }
$$

where $U_{C P} \nu_{\alpha L} U_{C P}^{-1}=\nu_{\alpha R}^{c}$ and $M_{\alpha \beta}$ is a symmetric matrix. For $C P$ invariance it is also real and can be diagonalized by an orthogonal matrix

$$
O_{i \alpha} M_{\alpha \beta} O_{\beta j}^{T}=\eta_{i} m_{i} \delta_{i j}
$$

with $\eta_{i}= \pm 1$ and $m_{i} \geq 0$. Choosing $\chi_{i}=\sum_{a} O_{i \alpha} \nu_{\alpha L}+\eta_{i} O_{i \alpha}\left(\nu_{\alpha L}\right)^{c}$ we have $-2 \mathcal{L}_{\text {mass }}=$ $m_{i} \bar{\chi}_{i} \chi_{i}$ and $U_{C P} \chi_{i} U_{C P}^{-1}=\eta_{i} \chi_{i}$. For $C P$ invariance it can be shown [21] that $\eta_{i}$ is connected to the intrinsic $C P$ parity of the Majorana, i.e. $\eta_{i}=i \eta_{i}^{C P}$. In addition it holds $\chi^{c}= \pm \eta_{i} \chi$. Therefore the $0 \nu \beta \beta$ amplitude for $C P$ invariance is proportional to [22]

$$
\mathcal{M}_{C P}(0 \nu \beta \beta) \propto O_{e i}^{2} \gamma_{-} \chi_{i} \overline{\chi_{i}^{c}} \gamma_{-} \propto \eta_{i} m_{i} O_{e i}^{2},
$$

with $2 \gamma_{ \pm}=1 \pm \gamma_{5}$. At the cost of a complex $O$ the $C P$ parities can also be absorbed in the mixing matrix via the identity $\eta_{i}=e^{i \pi / 2\left(\eta_{i}-1\right)}$. For complex $M_{\alpha \beta}$ and thus $C P$ violation" the diagonalization of the mass term is done by an unitary matrix,

$$
U_{i \alpha} M_{\alpha \beta} U_{\beta j}^{T}=m_{i} \delta_{i j} .
$$

Appropriate choice of $U$ can always make $m_{i} \geq 0$. Here, $\eta_{i}=1$ and the $0 \nu \beta \beta$ amplitude is proportional to

$$
\mathcal{M}_{C p}(0 \nu \beta \beta) \propto U_{e i}^{2} \gamma_{-} \chi_{i} \overline{\chi_{i}^{c}} \gamma_{-} \propto m_{i} U_{e i}^{2} .
$$

\footnotetext{
${ }^{1} \mathrm{~A}$ geometrical description of $C P$ violation with Majorana neutrinos in terms of unitarity triangles can be found in 23].
} 
To conclude, the matrix in the relation $\nu_{\alpha}=S_{\alpha i} \nu_{i}$ is either orthogonal or unitarity and the quantity probed in $0 \nu \beta \beta$ is

$$
\langle m\rangle=\left\{\begin{array}{cc}
\left|\sum_{i} U_{e i}^{2} m_{i}\right| & C P \text { violation } \\
\left|\sum_{i} O_{e i}^{2} m_{i} \eta_{i}\right| & C P \text { invariance }
\end{array} .\right.
$$

For 2 flavors the two cases read:

$$
\langle m\rangle=\left\{\begin{array}{lc}
\left|m_{1} \cos ^{2} \theta+m_{2} \sin ^{2} \theta e^{2 i \phi}\right| & C P \text { violation } \\
\left|m_{1} \cos ^{2} \theta+m_{2} \eta_{1} \eta_{2} \sin ^{2} \theta\right| & C P \text { invariance }
\end{array} .\right.
$$

Hence, a single $C P$ parity has no physical meaning, only relative values are significant. Note though that the case $\eta_{1} \eta_{2}=-1$ (opposite parities) and $\phi=\pi / 2$ ("maximal" violation) can not be distinguished. This can be also seen from the choice of the mixing matrix $S_{\alpha i}$ : The case $\eta_{1}=-1$ and $S_{e 1}=O_{e 1}$ is equivalent to $\eta_{1}=+1$ and $S_{e 1}=e^{-i \pi / 2} O_{e 1}=-i O_{e 1}$. This means that opposite parities with a real mixing matrix are equivalent to equal parities with a complex mixing matrix for a maximal phase. Hence, for "maximal" phases we can not tell from $0 \nu \beta \beta$ alone if there is $C P$ violation in the lepton sector and the answer if there is one at all has to come from long-baseline experiments [24]. This is similar to the statement by Pal and Wolfenstein 25] for the decay $\nu_{2} \rightarrow \nu_{1} \gamma$ : For equal (opposite) parities magnetic (electric) dipole radiation occurs. Opposite parities are equivalent to complex mixing matrix elements but do not imply $C P$ violation. This would be suggested only by the presence of both kinds of radiation. For a degenerate scheme with masses discussed in the following sections however, the life time of such a process is about $\tau \gtrsim 10^{48} \mathrm{~s}$.

\section{Oscillation Experiments, $0 \nu \beta \beta$ and $\langle m\rangle$ in Hierar- chical Schemes}

The $C P$-violating mixing matrix $U$ can be parametrized as

$$
\begin{gathered}
U=U_{\mathrm{CKM}} \operatorname{diag}\left(1, e^{i \alpha}, e^{i(\beta+\delta)}\right) \\
=\left(\begin{array}{ccc}
c_{1} c_{3} & s_{1} c_{3} & s_{3} e^{-i \delta} \\
-s_{1} c_{2}-c_{1} s_{2} s_{3} e^{i \delta} & c_{1} c_{2}-s_{1} s_{2} s_{3} e^{i \delta} & s_{2} c_{3} \\
s_{1} s_{2}-c_{1} c_{2} s_{3} e^{i \delta} & -c_{1} s_{2}-s_{1} c_{2} s_{3} e^{i \delta} & c_{2} c_{3}
\end{array}\right) \operatorname{diag}\left(1, e^{i \alpha}, e^{i(\beta+\delta)}\right),
\end{gathered}
$$

where $c_{i}=\cos \theta_{i}$ and $s_{i}=\sin \theta_{i}$. The orthogonal matrix $O$ is of course obtained by setting the phases to zero. From hereon we will always write $U_{\alpha i}$ for the mixing matrix with obvious changes for the $C P$ conserving case. Since the oscillation probability

$$
P_{\alpha \beta}=\delta_{\alpha \beta}-2 \operatorname{Re} \sum_{j>i} U_{\alpha i} U_{\alpha j}^{*} U_{\beta i}^{*} U_{\beta j}\left(1-\exp i \Delta_{j i}\right) .
$$


is invariant under such a multiplication of a diagonal matrix, the two additional Majorana induced phases are not observable in any oscillation experiment [26]. Therefore, $0 \nu \beta \beta$ is the only probe to test these phases. In principle it would be possible to completely derive all phases by comparing the elements of the Majorana mass matrix

$$
\left\langle m_{\alpha \beta}\right\rangle=\left|\left(U \operatorname{diag}\left(m_{1} \eta_{1}, m_{2} \eta_{2}, m_{3} \eta_{3}\right) U^{\mathrm{T}}\right)_{\alpha \beta}\right| .
$$

However, the analogues of $0 \nu \beta \beta$ one needs to observe like $K^{+} \rightarrow \pi^{-} \mu^{+} \mu^{+}$[27], $\nu_{\mu} N \rightarrow$ $\mu^{-} \mu^{+} \mu^{+} X$ [28] or $e^{+} p \rightarrow \bar{\nu}_{e} \mu^{+} \tau^{+} X$ [29] have branching ratios or cross section far too small to be measured [14].

All numerical analyses indicate a hierarchy in the mass squared differences of solar and atmospheric experiments:

$$
\Delta m_{\odot}^{2} \ll \Delta m_{A}^{2} .
$$

Maximal values are $\left(\Delta m_{\odot}^{2}\right)_{\max } \simeq 10^{-4} \mathrm{eV}^{2}$ 30, 31] and $\left(\Delta m_{A}^{2}\right)_{\max } \simeq 10^{-2} \mathrm{eV}^{2}$ [32]. Without loss of generality we assume $m_{3} \geq m_{2} \geq m_{1}$. In a 3 flavor picture, three hierarchical schemes, where at least for one mass eigenstate $m_{i}^{2} \simeq \Delta m^{2}$ holds, are capable of explaining the relation (12), they are called "completely hierarchical" $\left(m_{3} \simeq \sqrt{\Delta m_{A}^{2}} \gg m_{2} \simeq\right.$ $\left.\sqrt{\Delta m_{\odot}^{2}} \gg m_{1}\right)$, "partially hierarchical" $\left(m_{3} \simeq \sqrt{\Delta m_{A}^{2}} \gg m_{2} \simeq m_{1}\right)$ and "inverse hierarchical" $\left(m_{3} \simeq m_{2}=\sqrt{\Delta m_{A}^{2}} \gg m_{1}\right)$. With this notation, the CHOOZ result demands a small $\left|U_{e 3}\right|$ in the first two cases and a small $\left|U_{e 1}\right|$ in the last one. The latter fact stems from the condition that for $m_{3} \geq m_{2} \geq m_{1}$ the completely and partially hierarchical schemes demand $\Delta m_{21}^{2}=\Delta m_{\odot}^{2} \ll \Delta m_{31}^{2} \simeq \Delta m_{32}^{2}$, whereas the inverse hierarchical scheme has to be given by $\Delta m_{32}^{2}=\Delta m_{\odot}^{2} \ll \Delta m_{21}^{2} \simeq \Delta m_{31}^{2}$. With a good approximation we can use 2 flavor fits, then the solar mixing angle gives

$$
c_{1}^{2}, s_{1}^{2} \simeq \frac{1}{2}\left(1 \pm \sqrt{1-\sin ^{2} 2 \theta_{\odot}}\right) .
$$

The data we use are taken from Refs. [30, 31] and given in Table 1. As usual, SA (LA) denotes the small (large) angle MSW, LOW the MSW low mass and VO the vacuum solution. The MSW [33] resonance condition demands $\left|U_{e 1}\right| \geq\left|U_{e 2}\right|$. However, for the vacuum solution this might not be the case, we come back later on that point. In the inverse hierarchical scheme the resonance requires $\left|U_{e 3}\right| \geq\left|U_{e 2}\right|$. The CHOOZ experiment [34] gives unfortunately only a limit on $\left|U_{e 3}\right|$ (in the inverse hierarchical scheme on $\left|U_{e 1}\right|$ ), depending on the atmospheric mass scale, it reads at $90 \% \mathrm{C}$. L.

$$
\left|U_{e 3}\right|^{2} \lesssim 0.01 \ldots 0.15 \text { for } \Delta m_{A}^{2} \simeq 10^{-3} \ldots 10^{-2} \mathrm{eV}^{2}[35]
$$

For typical best-fit values of few $\times 10^{-3} \mathrm{eV}$ the bound is about $\left|U_{e 3}\right|^{2} \lesssim 0.05$. Even for the maximal allowed values of the mass squared differences and mixing angles $\langle m\rangle$ is always 
below $0.2 \mathrm{eV}$ :

$$
\begin{aligned}
& \langle m\rangle \leq m_{1}\left|U_{e 1}\right|^{2}+m_{2}\left|U_{e 2}\right|^{2}+m_{3}\left|U_{e 3}\right|^{2} \\
& \leq\left\{\begin{array}{ccc}
m_{1}+\left|U_{e 2}\right|^{2} \sqrt{\Delta m_{\odot}^{2}}+\left|U_{e 3}\right|^{2} \sqrt{\Delta m_{A}^{2}} & \leq m_{1}+0.022 & \text { completely hierarchical } \\
\left(\left|U_{e 1}\right|^{2}+\left|U_{e 2}\right|^{2}\right) \sqrt{\Delta m_{\odot}^{2}}+\left|U_{e 3}\right|^{2} \sqrt{\Delta m_{A}^{2}} & \leq 0.85 m_{1}+0.015 & \text { partially hierarchical } \\
\left|U_{e 1}\right|^{2} m_{1}+\left(1-\left|U_{e 1}\right|^{2}\right) \sqrt{\Delta m_{A}^{2}} & \leq m_{1}+0.085 & \text { inverse hierarchical }
\end{array} .\right.
\end{aligned}
$$

Note that there can be a additional contribution of $m_{1}<\sqrt{\Delta m_{\odot}^{2}}$, which can be traced back to the fact that only mass squared differences are measured. If an experimental sensitivity of $10^{-2} \mathrm{eV}$ on $\langle m\rangle$ is achieved, this might be of importance. However, at the present time with $\langle m\rangle \leq 0.2 \mathrm{eV}$, all values of the phases and the parities are allowed. In order to make more definite statements about the effects of cancellations, we therefore apply a degenerate scheme in which $m_{3}^{2} \simeq m_{2}^{2} \simeq m_{1}^{2} \equiv m_{0}^{2}$. Per definition, it should hold $m_{0}^{2} \gg \Delta m_{\max }^{2} \simeq 10^{-2}$. When in the following a maximal allowed mass scale of $m_{0} \simeq 0.2 \ldots 0.3 \mathrm{eV}$ is derived so does that mean that the mass scheme is not "completely degenerate" but "slightly" hierarchical, i.e. an intermediate situation occurs. For example, if $m_{0, \max }=0.2 \mathrm{eV}$, then another eigenstate has a mass of $(0.2-\varepsilon) \mathrm{eV}$, where $\varepsilon$ is small. In order to get the atmospheric scale of $10^{-2} \mathrm{eV}^{2}$ one finds $\varepsilon \simeq 0.03 \mathrm{eV}$ and the two eigenstates differ by about $15 \%$. Then our $m_{0, \max }$ is the limit on the largest mass eigenstate $m_{3}$ with $m_{3} \simeq$ or $>m_{2}$. A limit of $m_{0} \simeq 0.1 \mathrm{eV}$ corresponds to a hierarchical scheme. However, due to the uncertainty in the value of $\langle m\rangle$ and $\Delta m_{A}^{2}$ a definite statement is somehow difficult to make. Using the best-fit point for the atmospheric scale means that the degenerate scheme fails for about $0.1 \mathrm{eV}$.

\section{$4\langle m\rangle$ in the Degenerate Scheme}

The upper bound on $m_{0}$ comes from the tritium spectrum, which limits [36]

$$
\sum_{i}\left|U_{e i}^{2}\right| m_{i}^{2}=m_{0}^{2} \leq(2.8 \mathrm{eV})^{2}
$$

Forthcoming and ongoing projects intend to push the bound below $1 \mathrm{eV}$ [37]. In addition, cosmological observations might be interpreted in terms of a total neutrino mass of $\sum m_{\nu} \simeq$ few $\mathrm{eV}$ [38]. By measuring anisotropies in the cosmic microwave background, MAP and PLANCK may probe values down to $\sum m_{\nu} \sim 0.5 \mathrm{eV}$ [39]. The interesting function to investigate is $\langle m\rangle / m_{0} \equiv \tilde{m}$, which is depending on 4 parameters, either two angles and 2 phases or two angles and two relative parities. In the $C P$-violating case $\tilde{m}$ reads

$$
\frac{\langle m\rangle}{m_{0}} \equiv \tilde{m}=c_{3}^{2} \sqrt{c_{1}^{4}+s_{1}^{4}+t_{3}^{4}+2\left(s_{1}^{2} t_{3}^{2} c_{2(\alpha-\beta)}+c_{1}^{2}\left(s_{1}^{2} c_{2 \alpha}+t_{3}^{2} c_{2 \beta}\right)\right)}
$$


where $t_{3}=\tan \theta_{3}$ and $c_{2 \alpha}=\cos 2 \alpha$. For $C P$ invariance it can be written as

$$
\tilde{m}=c_{3}^{2}\left(\eta_{1} c_{1}^{2}+\eta_{2} s_{1}^{2}+\eta_{3} t_{3}^{2}\right)=\left\{\begin{array}{ccc}
1 & (+++) & \leftrightarrow \alpha=\beta=\pi \\
\left|c_{3}^{2}\left(c_{1}^{2}-s_{1}^{2}-t_{3}^{2}\right)\right| & (+--) & \leftrightarrow \alpha=\beta=\frac{\pi}{2} \\
\left|c_{3}^{2}\left(c_{1}^{2}-s_{1}^{2}+t_{3}^{2}\right)\right| & (-+-) & \leftrightarrow \alpha=\frac{\beta}{2}=\frac{\pi}{2} \\
\cos 2 \theta_{3} & (--+) & \leftrightarrow \alpha=2 \beta=\pi
\end{array},\right.
$$

where all 4 possible $( \pm \pm \pm)$ signatures with the corresponding $C P$-violating phases are given. In addition, there are $\left(c_{1}\right.$-dependent) solutions for the phases, which give the same $\tilde{m}$ as special parity configurations, see below. Note that for $(--+)$ the value is independent on the solar solution. The $(+--)$ signature is in fact the minimal value for the general $C P$-violating case, provided that $\left|U_{e 1}\right| \geq\left|U_{e 2}\right|$. For the inverse situation, the $(-+-)$ signature gives the minimal $\tilde{m}$.

The different treatment of $C P$ parities and phases may seem somewhat artificial since the first option is a special case of the latter one. However, due to the maximal values certain configurations result in they deserve special attention.

\subsection{Some special mixing matrices}

Three intriguingly simple matrices have been widely discussed:

- Single maximal [40] Maximal atmospheric mixing and a vanishing angle in solar and the CHOOZ experiment, resulting in $\left|U_{e 1}\right|=1$.

- Bimaximal 41

Both solar and atmospheric mixing is maximal, CHOOZ's angle is zero. Then $\left|U_{e 1}\right|=$ $\left|U_{e 2}\right|=1 / \sqrt{2}$ and $\left|U_{e 3}\right|=0$.

- Trimaximal [42

All elements have the same magnitude and $\left|U_{e 1}\right|=\left|U_{e 2}\right|=\left|U_{e 3}\right|=1 / \sqrt{3}$. The model gives a poor fit to the oscillation data.

Then one gets for the $C P-$ conserving case

$$
\tilde{m}= \begin{cases}1,1,1 & (+++) \\ 1,0, \frac{1}{3} & (+--) \\ 1,0, \frac{1}{3} & (-+-) \\ 1,1, \frac{1}{3} & (--+)\end{cases}
$$

for single, bi- and trimaximal mixing respectively. Single and bimaximal mixing are special cases of the mixing angles from Table 1 and might serve as a model to get a feeling for 
the bounds one obtains. With the current limit on $\langle m\rangle$ we get for values of $\tilde{m}=1, \frac{1}{3}$ : $m_{0} \leq 0.2,0.6$. Obviously, for $\tilde{m}=0$ it follows $\langle m\rangle=0$. If there is $C P$ violation we have

$$
\tilde{m}=\left\{\begin{array}{cc}
1 & \text { single } \\
c_{\alpha} & \text { bi } \\
\frac{1}{3} \sqrt{3+2\left(c_{2 \alpha}+c_{2 \beta}+c_{2(\alpha-\beta)}\right)} & \text { tri }
\end{array} .\right.
$$

Single maximal mixing means $m_{0} \leq 0.2 \mathrm{eV}$, unobtainable by currently planned experiments. For bimaximal mixing we get with the current limit and assuming $m_{0}=1 \mathrm{eV}$ : $\alpha \lesssim 78.5^{0}$. For the trimaximal case Fig. 1 shows $\tilde{m}$ as a function of one phase for different values of the second phase. Note the constant value for $\beta=\pi / 2$.

\subsection{General Limits and Bounds}

We go now from the special cases back to all experimentally allowed values of the mixing angels. The first thing to say is that for exactly vanishing $U_{e 3}$ there is no way to find out about the second phase ${ }^{2}$. Without further effort we can say for the $(+++)$ signature, that $m_{0} \leq 0.2$, i.e. is beyond experimental access in cosmology or spectrum measurements. More interesting is e.g. the $(--+)$ case in which $\tilde{m}$ is depending on only one parameter, namely the angle bounded by CHOOZ. With Eqs. (11) and (14) we get the allowed range of $m_{0}$ depicted in Fig. 2. In this situation, the maximal neutrino mass is about $0.29 \mathrm{eV}$, using the best-fit point of SK gives $m_{0} \leq 0.22 \mathrm{eV}$. We also plot the range for a limit of $\langle m\rangle<0.1 \mathrm{eV}$, which further reduces the allowed values. The maximal $m_{0}$ is now 0.14 and $0.11 \mathrm{eV}$, respectively. As can be seen, if cosmology insists in $m_{0} \simeq 1 \mathrm{eV}$, the $(--+)$ configuration is ruled out.

There is still freedom in the ordering of $\left|U_{e 1}\right|$ and $\left|U_{e 2}\right|$ if the vacuum solution is correct. We denote the choice $\left|U_{e 1}\right|>\left|U_{e 2}\right|$ with VO1 and the other one with VO2. However, as can be seen from Eqs. (13) and (18) the case VO1 and $(+--)$ is equivalent to VO2 and $(-+-)$ as VO1 and $(-+-)$ is to VO2 and $(+--)$. In Figures 3 and 1 we show the maximal values of $m_{0}$ as a function of $\left|U_{e 3}\right|^{2}$ for the current $\langle m\rangle$-limit of $0.2 \mathrm{eV}$. These maximal values scale with the $\langle m\rangle$ limit. In [10] plots of the allowed $\left|U_{e 1}\right|^{2}$ and $\left|U_{e 3}\right|^{2}$ were given for $m_{0}=1.7 \mathrm{eV}$ and all 3 nontrivial parity configurations. Their conclusion that small $\left|U_{e 3}\right|$ requires near-maximal mixing for the $(-+-)$ and $(+--)$ case is consistent with Figs. 3 and 4 . For $(--+)$ they find that only small $m_{0}$ allows large mixing, which can be seen in Fig. $⿴$ as well. The authors also give the allowed areas in $\left|U_{e 1}\right|^{2}-\left|U_{e 3}\right|^{2}$ space for special values of the phases. However, to obtain these areas they included all $( \pm \pm \pm)$ possibilities, which is hard to compare with our approach. Reference [8] also plots $\left|U_{e 1}\right|^{2}$ against $\left|U_{e 3}\right|^{2}$ for different situations resulting in similar conclusions as ours. Our plots are thus different projections of the 5 dimensional parameter space giving complementary and additional information. Both mentioned works use cosmological arguments to set $m_{0}$ to 1.7 to $4 \mathrm{eV}$, whereas our approach (also used in [17]) allows to investigate different situations

\footnotetext{
${ }^{2}$ Also, "normal" $C P$ violation in long-baseline experiments will be unobservable.
} 
like positive results on $\langle m\rangle$ and/or $m_{0}$.

In Figs. 5 and 0 we plot $\tilde{m}$ as a function of $\alpha$ for different values of $\beta$ whilst assuming $\left|U_{e 3}\right|^{2}=0.03$. Maximal mixing (i.e. $\left|U_{e 1}\right|=\left|U_{e 2}\right|$ ) allows complete cancellation, whereas for other values a non-vanishing minimal $\tilde{m}$ is achieved. The dependence on the second phase is rather small which can be explained by the smallness of $\left|U_{e 3}\right|$, confer with Fig. 1, where the dependence on the second phase is rather strong. In Fig. 7 we therefore show for $\langle m\rangle \leq 0.2 \mathrm{eV}, \beta=\pi / 2$ and $\left|U_{e 3}\right|^{2}=0.03$ the allowed area in the $m_{0}-\alpha$ space for different $\sin ^{2} 2 \theta_{\odot}$. The higher this angle is, the higher is the maximal allowed $m_{0}$. This value is approached for a solar mixing angle of about 0.7 , which is also true for the $(+--)$ and $(-+-)$ configurations. For the SA solution it follows $c_{1} \simeq 1$ in Eq. (18) so that $\tilde{m}$ is always about 1 . This means if the SA solution turns out to be realized in nature direct mass searches will not find the neutrino mass scale because eutrinoless double beta decay then already demands $m_{0} \leq 0.2 \mathrm{eV}$. If $m_{0} \gtrsim 0.5 \mathrm{eV}$ is measured and SA turns out to be true, then neutrinos are Dirac particles. These are well known facts and are only given for the sake of completeness.

The best-fit value and the minimal respectively maximal allowed mixing angle gives lines too close together to be distinguishable in the plot. Regarding VO1 and VO2 it turns out that the difference for given phases is negligible (1 to $2 \%$ ), so we plot in Fig. 6 only the VO1 option. The difference between the two options would only be sizable for large $\left|U_{e 3}\right|$. If the bounds on $\langle m\rangle$ and $m_{0}$ are both further reduced, nothing special happens. Some more interesting possibilities however suggest themselves: First, a value for $m_{0}$ is found and $\langle m\rangle$ continues to give an upper bound. Then we get a lower limit on $\tilde{m}$. Second, the reverse situation results in an upper limit on $\tilde{m}$. If both quantities are measured, then definite statements about the phases can be made. In connection with the then already obtained knowledge of the correct solar solution and a more stringent $\mathrm{CHOOZ}$ bound (or signal) some important conclusions could be drawn. In addition, we can assume that the precision in the mixing angles will be improved. To simplify things a bit we take now the best-fit values. Since these points of the VO and the LA solution are very close together we only plot the LA and the LOW case in Figs. 8 and 9, respectively. Recent data of the day-night spectrum seems to favor these solutions [43]. Different values of the parameters lead to modifications of the results which are not difficult to do with the help of Eqs. (13, 17, 18). From the figures, we can easily analyze some specific configurations: For example, for $\tilde{m}<0.6$ the $(--+)$ configuration is ruled out and for $\tilde{m}>0.6$ the $(+--)$ and $(-+-)$ cases, making 0.6 a key scale, since it is independent on the solar solution and the value of $\left|U_{e 3}\right|$. If we would know the value of $\tilde{m}$, even more could be said: The key value of 0.6 would mean that there is $C P$ violation in the leptonic sector. With the current limits on $\langle m\rangle$ and $m_{0}$ and the maximal sensitivities achievable by current experiments, only $\tilde{m}<0.6$ seems realistic. The highest value with currently planned experiments is in fact $\tilde{m}=0.2 / 0.5=0.4$, which for small $\left|U_{e 3}\right|$ would rule out $(+--)$ and $(-+-)$ for LOW. Also, again for small $\left|U_{e 3}\right|$, the LA and VO solution would be out of the game. Note however that the mentioned uncertainty for the nuclear matrix elements might allow to use a higher value for $\langle m\rangle$, namely about $\sqrt{3}\langle m\rangle$ which then might allow to reach a $\tilde{m}$ value of 0.6 . 
Conversely, the smallest number one can expect to measure is $\tilde{m}=2 \cdot 10^{-3} / 2.8=7$. $10^{-4}$, which forbids the degenerate scheme at all and demands hierarchical or intermediate schemes. We refer to Refs. [16, 17] for the allowed values of $\langle m\rangle$ in that case. In general, as can be seen from the Figures, a value of $\tilde{m}$ below $0.24(0.06)$ rules out the degenerate scheme for the LA and VO (LOW) solution. Alternatively, if LA (LOW) turns out to be true and a $\tilde{m}$ bound of 0.24 (0.06) is achieved, neutrinos are Dirac particles. Note however that our discussion relies on precise knowledge of the mixing angles and $\tilde{m}$ and has thus to be taken with care. A realistic assumption about the minimal uncertainty in reach for these measurements might be given by $20 \%$ for the former and a factor of 2 for the latter. We will show in the next section that the uncertainty for the other mass matrix elements is at least of this order.

\subsection{Consequences for the Mass Matrix}

We want to show that the uncertainty in the other mass matrix entries is at least as large as the one stemming from $m_{0}$, nuclear matrix elements and the solar mixing angle. To show this we shall assume that both quantities as well as $\left|U_{e 3}\right|$ and the angle governing the atmospheric neutrino anomaly are known precisely. As an example we take $\tilde{m}=0.3$ and $\left|U_{e 3}\right|^{2}=0.1$. This might be measured by $\langle m\rangle=0.18 \mathrm{eV}$ and $m_{0}=0.6 \mathrm{eV}$. However, different choice of the 2 phases can result in the same $\tilde{m}$, as can be seen in Fig. 10 for the LOW case, where "iso- $\tilde{m}$ " lines are displayed. For example, $(\alpha \simeq 1.177, \beta \simeq 1.864)$ or $(\alpha \simeq 1.7691, \beta \simeq 0.5225)$ are possible solutions for $\tilde{m}=0.3$. The $\mu \mu$ entry of the mass matrix, $m_{\mu \mu}=m_{0}\left|\left(U_{\mu 1}^{2}+U_{\mu 2}^{2}+U_{\mu 3}^{2}\right)\right|$ is still a function of the third phase $\delta$. Assuming maximal atmospheric mixing, the two results for $m_{\mu \mu}$ differ by a factor of 4 (for $\delta=0$ ) to 8 for $(\delta \simeq 2.8)$. It turns out that for a given $\delta$ some specific choices of the other two phases give for the resulting $m_{\mu \mu}$ relative differences of up to 15. Thus, to verify the real solution, one would need a measurement of $m_{\mu \mu}$. The charged kaon decay $K^{+} \rightarrow \pi^{-} \mu^{+} \mu^{+}$, which is depending on $m_{\mu \mu}$ as $0 \nu \beta \beta$ is on $\langle m\rangle$, has a branching ratio given by

$$
\frac{\Gamma\left(K^{+} \rightarrow \pi^{-} \mu^{+} \mu^{+}\right)}{\Gamma\left(K^{+} \rightarrow \text { all }\right)} \simeq 10^{-31}\left(\frac{m_{\mu \mu}}{\mathrm{eV}}\right)^{2} \lesssim 8 \cdot 10^{-31}
$$

which has to be compared with the experimental limit of $3 \times 10^{-9}$ [44. Even worse numbers hold for the other elements of the mass matrices and the respective processes they govern, see [14 for a compilation. The closer the measured $\tilde{m}$ value gets to the minimal value (0.1205 for our specific example) the smaller gets the allowed area or curve in $\alpha-\beta$ space and therefore the resulting range for the other matrix elements. This minimal value of $\tilde{m}$ corresponds to the $(+--)$ configuration. Of course, also for the maximal value of $\tilde{m}=1$ only one pair of phases is responsible, however, then holds $m_{0} \leq 0.2 \mathrm{eV}$. For a precision of $10 \%$ in the measurement of the minimal $\tilde{m}$ we get a variation in $m_{\mu \mu}$ within a factor of 2 . A slightly smaller number is obtained for a $5 \%$ precision. In Fig. 10 the area for $\tilde{m}=0.1205$ is obtained for a precision of $5 \%$. However, it seems to be questionable that these values of $\tilde{m}$ and the required precision are feasible, especially in the light of the different results 
of different nuclear matrix element calculations. Thus one needs to make compromises in terms of theoretical assumptions [45] in order to get the complete mass matrix. However, despite the large uncertainty in the other mass matrix entries, note that in most cases the mass spectrum of the eigenvalues can still be probed: from Fig. 26 of Ref. [16] it is seen that - provided the solar solution is known - some measured values of $\langle m\rangle$ distinguish different schemes. The many possibilities for $\alpha$ and $\beta$ can be understood in the following way: In a three flavor scheme we have 9 parameters: 3 masses, 3 angles and 3 phases. Oscillation experiments can give two mass squared differences (equivalent to 2 masses), all 3 angles and 1 phase. The mass scale might be given by the tritium spectrum or from cosmology, so that we are left with 2 parameters and one observable, namely $\langle m\rangle$. Only for minimal $\tilde{m}$ an unambiguous determination of the 2 phases is possible, which however requires an extremely precise measurement of this quantity. The smallness of $\left|U_{e 3}\right|$ allows a broad range for $\beta$, but for the $\mu \mu$ entry $\beta$ contributes with $c_{2} c_{3} e^{i \beta} \simeq 1 / \sqrt{2} e^{i \beta}$, which can have a large effect on the magnitude of $m_{\mu \mu}$.

To conclude, for the $(+--)$ case the uncertainty in $m_{\mu \mu}$ is at least of the same order as the one stemming from the solar angles and $\tilde{m}$. In general the difference can be up to factors around 20 .

If we choose a smaller $\left|U_{e 3}\right|$ then the uncertainty in $\beta$ is even larger and our arguments are strengthened. For $m_{0}$ smaller then $0.3 \mathrm{eV}$, Eq.(15) tells us that $\langle m\rangle \lesssim m_{1}$, which is the smallest eigenvalue, lying at the very end of the planned $\langle m\rangle$ sensitivity. An analysis of the phases is then probably impossible and again our arguments are strengthened.

\section{Conclusions}

In a degenerate mass scheme with three neutrinos we did a full analysis of the allowed parameter range for the relevant function $\tilde{m}=\langle m\rangle / m_{0}$. All $C P$-conserving and -violating possibilities for cancellation were considered and plots for observables in each case were given. When $m_{0, \max }$ is smaller then roughly $0.2 \ldots 0.1 \mathrm{eV}$, the limit applies no longer to a degenerate scheme but for the highest mass of a hierarchical or intermediate scheme. The other masses are then given by the measured mass squared differences. Then however a signal or improved bound from $0 \nu \beta \beta$ is needed to distinguish the different possibilities, i.e. to verify which two masses give which $\Delta m^{2}$. For the SA solution the mass scale is below $0.2 \mathrm{eV}$, regardless of $C P$ violation or conservation. Effects of phases then lie in the $\%$ range. We may summarize the situation for $C P$ invariance such that the $(+++)$ case also means $m_{0} \leq 0.2 \mathrm{eV}$. If the $(--+)$ configuration is realized (Fig. 2), the total mass scale is too small to be directly measured. Regarding the other two parity configurations (Figs. 3 and 4), maximal solar mixing allows a broad range for $m_{0}$, for small $\left|U_{e 3}\right|^{2}$ even up to the tritium limit of $2.8 \mathrm{eV}$. The smaller the angle $\sin ^{2} 2 \theta_{\odot}$ is, the smaller is the maximal allowed $m_{0}$ or the larger is the minimal value of $\tilde{m}$. For $C P$ violation two phases are present, in principle varying between 0 and $\pi$. Because of the smallness of $\left|U_{e 3}\right|^{2}$ the dependence on one phase is small. For maximal solar mixing complete cancellation and therefore $m_{0}$ up to $2.8 \mathrm{eV}$ is possible. From $\sin ^{2} 2 \theta_{\odot} \simeq 0.7$ on, $m_{0, \text { max }}$ approaches $0.2 \mathrm{eV}$ 
and the degenerate scheme is jeopardized. A key value for $\tilde{m}$ of 0.6 was identified, which would prove the existence of $C P$ violation or would rule out some parity configurations. This value can unfortunately only be reached if we loosen the $\langle m\rangle$ bound with respect to the uncertainty in nuclear matrix element calculations. If we change the limit on $\langle m\rangle$ by a factor of $x$ then all the maximal allowed values for $m_{0}$ obtained here have to be multiplied with this very factor $x$.

Provided neutrinos are Majorana particles, pushing $\tilde{m}$ below 0.24 would rule out the LA and VO solution, and further reduction below 0.06 would rule out also the LOW case. If luckily the minimal $\tilde{m}$ would be measured, an uncertainty of $10 \%$ in $\tilde{m}$ translates into a variation of the other mass matrix entries of about a factor of 2. For non-minimal values many choices of the phases are possible. This will reflect on the result of the other entries in the mass matrices. The resulting uncertainty is as least as large as the one resulting from measurements of the solar angle and different calculations of nuclear matrix elements. Because the processes governed by these elements are way beyond experimental access, further input from the theoretical side is needed. Thus, with currently planned projects, experimental verification of a given mass matrix is very questionable.

\section{Acknowledgments}

I thank A. Joshipura, E. A. Paschos, W. G. Scott and L. Wolfenstein for helpful comments. This work has been supported in part by the "Bundesministerium für Bildung, Wissenschaft, Forschung und Technologie", Bonn under contract No. 05HT9PEA5. Financial support from the Graduate College "Erzeugung und Zerfälle von Elementarteilchen" at Dortmund university is gratefully acknowledged.

\section{References}

[1] For reviews see e.g. K. Zuber, Phys. Rep. 305, 295 (1998), S. M. Bilenky, C. Giunti, and W. Grimus, Prog. Nucl. Part. Phys. 43, 1 (1999).

[2] M. Gell-Mann, P. Ramond, and R. Slansky in Supergravity, p. 315, edited by F. Nieuwehuizen and D. Friedman, North Holland, Amsterdam, 1979; T. Yanagida, Proc. of the Workshop on Unified Theories and the Baryon Number of the Universe, edited by O. Sawada and A. Sugamoto, KEK, Japan 1979; R. N. Mohapatra, G. Senjanovic, Phys. Rev. Lett. 44, 912 (1980).

[3] M. Doi, T. Kotani, and E. Takasugi, Prog. Theo. Phys. Suppl. 83, 1 (1985), P. Vogel nucl-th/0005020.

[4] W. Rodejohann and K. Zuber, Phys. Rev. D 62, 094017 (2000).

[5] L. Baudis et al., Phys. Rev. Lett. 83, 41 (1999).

[6] H. V. Klapdor-Kleingrothaus et al., hep-ph/9910205. 
[7] S. T. Petcov and A. Yu. Smirnov, Phys. Lett. B 322, 109 (1994); S. M. Bilenky et al., Phys. Rev. D 54, 1881 (1996); Phys. Rev. D 57, 6981 (1998); Phys. Lett. B 465, 193 (1999).

[8] H. Minakata and O. Yasuda, Phys. Rev. D 56, 1692 (1997).

[9] T. Fukuyama, K. Matsuda and N. Nishura, Phys. Rev. D 57, 5844 (1998); Mod. Phys. Lett. A 13, 2279 (1998);

[10] R. Adhikari and G. Rajasekaran, Phys. Rev. D 61, 031301 (2000).

[11] M. Czakon, J. Gluza, and M. Zralek, Phys. Lett. B 465, 211 (1999); hep-ph/0003161.

[12] V. Barger and K. Whisnant, Phys. Lett. B 456, 194 (2000).

[13] F. Vissani, J. High Energy Phys. 06, 22 (1999).

[14] W. Rodejohann, Phys. Rev. D 62, 013011 (2000).

[15] T. Fukuyama et al., hep-ph/0003055.

[16] H. V. Klapdor-Kleingrothaus, H. Päs, and A. Yu. Smirnov, hep-ph/0003219.

[17] M. Czakon, J. Studnik, and M. Zralek, hep-ph/0006339.

[18] N. Haba et al., Prog. Theo. Phys. 103, 145 (2000); N. Haba, Y. Matsui, and N. Okamura, hep-ph/0005075; T. Miura et al., hep-ph/0005267.

[19] A. S. Joshipura and E. A. Paschos, hep-ph/9906498.

[20] L. Wolfenstein, Phys. Lett. B 107, 77 (1981); S. M. Bilenky, N. P. Nedelcheva, and S. T. Petcov, Nucl. Phys. B 247, 61 (1984).

[21] S. M. Bilenky and S. T. Petcov, Rev. Mod. Phys. 59, 671 (1983).

[22] B. Kayser, Phys. Rev. D 30, 1023 (1984).

[23] J. A. Aguilar-Saavedra and G. C. Branco, hep-ph/0007025; A. Donini, O, Mena, and S. Rigolin, hep-ph/0007281.

[24] See e.g. M. Masafumi and J. Sato, Phys. Rev. D 61, 073012 (2000).

[25] P. B. Pal and L. Wolfenstein, Phys. Rev. D 25, 766 (1982).

[26] S. M. Bilenky, J. Hosel, and S. T. Petcov, Phys. Lett. B 94, 495 (1980).

[27] A. Halprin et al., Phys. Rev. D 13, 2567 (1976); J. N. Ng and A. N. Kamal, Phys. Rev. D 18, 3412 (1978); J. Abad, J. G. Esteve, and A. F. Pachero, Phys. Rev. D 30, 1488 (1984); L. S. Littenberg and R. E. Shrock, Phys. Rev. Lett. 68, 443 (1992); hepph/0005285; K. Zuber, Phys. Lett. B 479, 33 (2000), C. Dib et al., hep-ph/0006277. 
[28] M. Flanz, W. Rodejohann, and K. Zuber, Eur. Phys. J. C 16, 453 (2000).

[29] M. Flanz, W. Rodejohann, and K. Zuber, Phys. Lett. B 473, 324 (2000), Phys. Lett. B 480, 418 (2000) (E).

[30] S. Goswami, D. Majumbar, and A. Raychauduri, hep-ph/9909453.

[31] M. C. Gonzalez-Garcia et al., Nucl. Phys. B 573, 3 (2000); M. C. Gonzalez-Garcia and C. Pena-Garay, Nucl. Phys. B (Proc. Suppl.) 87, 204 (2000).

[32] M. C. Gonzalez-Garcia, Nucl. Phys. B (Proc. Suppl.) 81, 113 (2000).

[33] L. Wolfenstein, Phys. Rev. D 17, 2369 (1978); S. P. Mikheyev and A. Yu. Smirnov, Yad. Fiz. 42, 1441 (1985).

[34] CHOOZ collaboration, M. Apollonio et al., Phys. Lett. B 466, 415 (1999).

[35] SuperKamiokande collaboration, Y. Fukuda et al., Phys. Rev. Lett. 81, 1562 (1998).

[36] C. Weinheimer et al., Phys. Lett. B 460, 219 (1999); V. M. Lobashev et al., Phys. Lett. B 460, 227 (1999).

[37] V. M. Lobashev, Prog. Nucl. Part. Phys. 40, 337 (1998).

[38] J. R. Primack and A. Klypin, Nucl. Phys. B (Proc. Suppl.) 51, 30 (1996).

[39] D. J. Eisenstein, W. Hu, and M. Tegmark, astro-ph/9807130.

[40] S. P. Rosen, W. Kwong, hep-ph/9501351.

[41] F. Vissani, hep-ph/9708483.

[42] L. Wolfenstein, Phys. Rev. D 18, 958 (1978); N. Cabibbo, Phys. Lett. B 72, 333 (1978); P. F. Harrison and W. G. Scott, Phys. Lett. B 333, 471 (1994); W. G. Scott, IDM98, 7-11 Sep 1998, Buxton UK. Proceedings by World Scientific, eds. N. J. C. Spooner and V. Kudryavtsev (1999) 540.

[43] SuperKamiokande collaboration, Y. Suzuki in Neutrino 2000, to appear, transparencies at http://nu2000.sno.laurentian.ca/Y.Suzuki/index.html

[44] E865 collaboration, H. Ma et al., hep-ex/0006003.

[45] For some recent speculations see e.g. D. Black et al. Phys. Rev. D 62, 073015 (2000); B. R. Desai, U. Sarkar, and A. R. Vaucher, hep-ph/0007346; L. Lavoura and W. Grimus, hep-ph/0008020; for reviews, see G. Altarelli and F. Feruglio, hep-ph/9905536; R. N. Mohapatra, hep-ph/9910365; S. M. Barr and I. Dorsner, hep-ph/0003058; C. H. Albright, hep-ph/0008013. 


\begin{tabular}{c|c|c} 
Solution & $\sin ^{2} 2 \theta_{\odot}(90 \%$ C.L. $)$ & Best-fit point \\
\hline VO [30] & $0.6 \ldots 1$ & 0.80 \\
\hline SA [31] & $7 \cdot 10^{-4} \ldots 10^{-2}$ & $5.5 \cdot 10^{-3}$ \\
\hline LA [31] & $0.55 \ldots 1$ & 0.79 \\
\hline LOW [31] & $0.75 \ldots 1$ & 0.94 \\
\hline
\end{tabular}

Table 1: Solution to the solar neutrino problem, 90 \% C. L. range of the mixing angle and best-fit point of the analysis. 


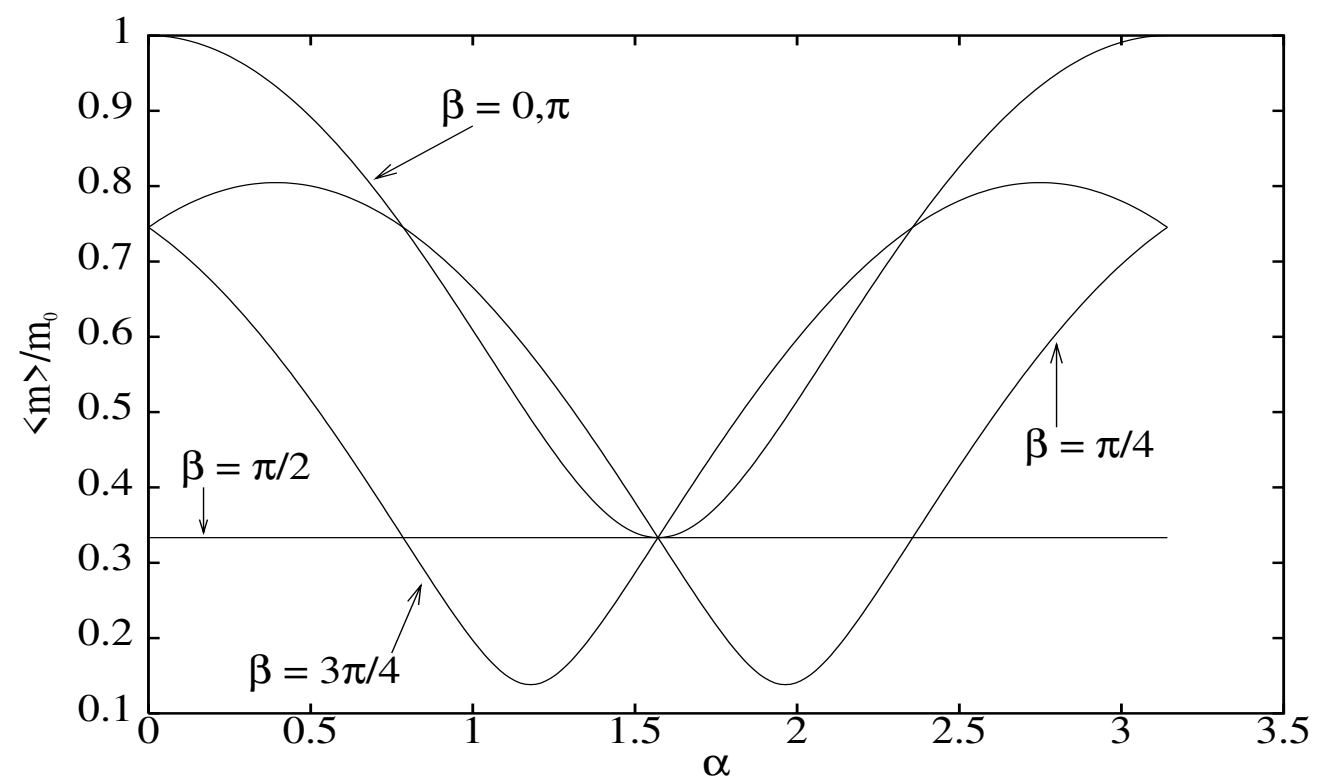

Figure 1: $\tilde{m}$ for trimaximal mixing as a function of one $C P$-violating phase for different values of the second phase.

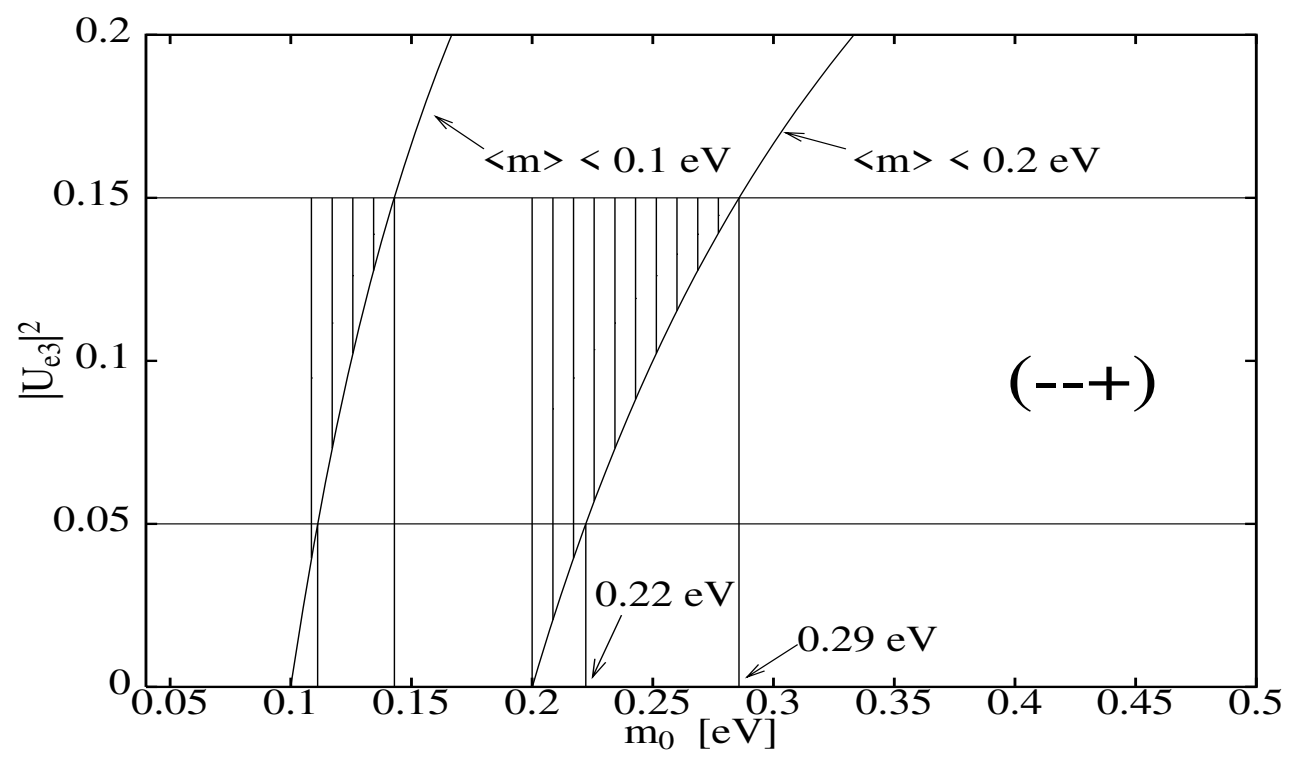

Figure 2: Allowed range for the common neutrino mass scale $m_{0}$ for $C P$ conservation and the $(--+)$ signature of the $C P$ parities. Displayed are the cases $\langle m\rangle \leq 0.2$ and $0.1 \mathrm{eV}$. The SK's best fit point corresponds to $m_{0}<0.22(0.11) \mathrm{eV}$ and the maximal value of $\left|U_{e 3}\right|^{2}$ leads to $m_{0}<0.29(0.14) \mathrm{eV}$ for the two values of $\langle m\rangle$. 


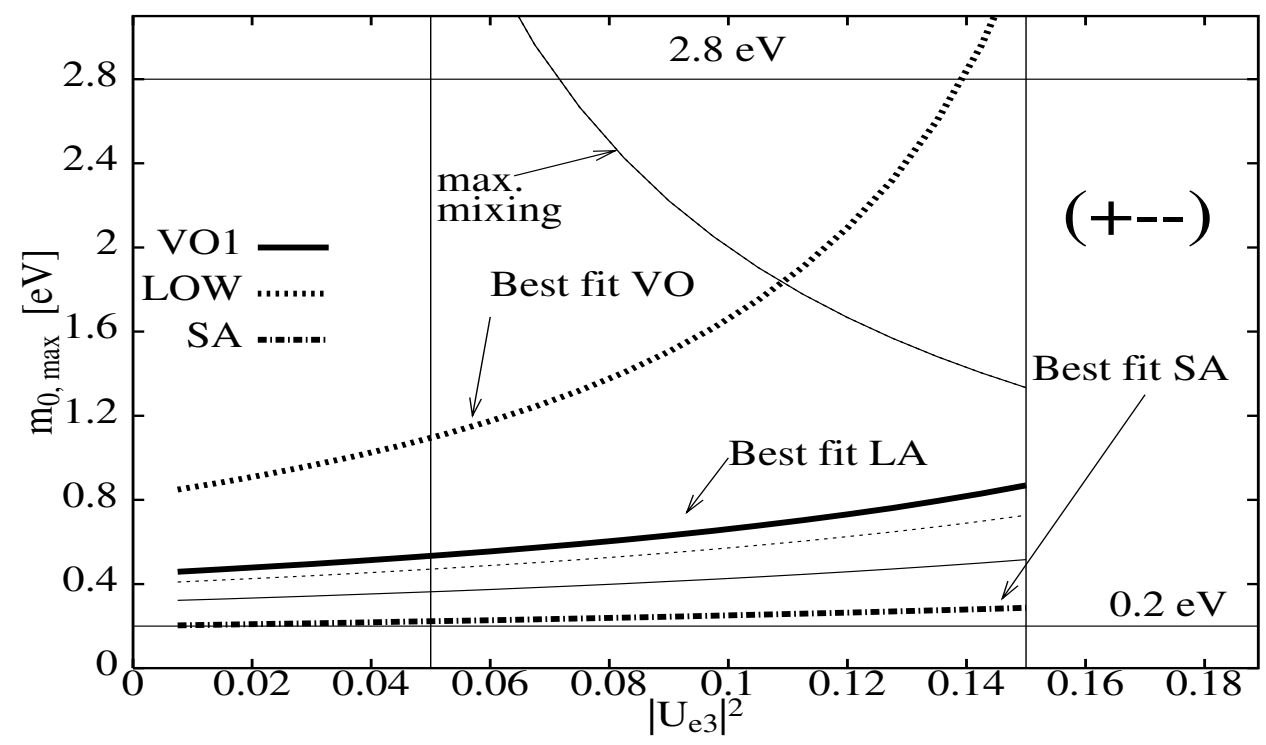

Figure 3: Maximal $m_{0}$ as a function of $\left|U_{e 3}\right|^{2}$ for the VO1, LOW and SA solution and the $(+--)$ signature. For LA the plot is very similar to VO1. A limit of $\langle m\rangle \leq 0.2 \mathrm{eV}$ is assumed. Allowed is the range under the respective curve. The VO2 option of the $(-+-)$ signature is the same as the VO1 option here.

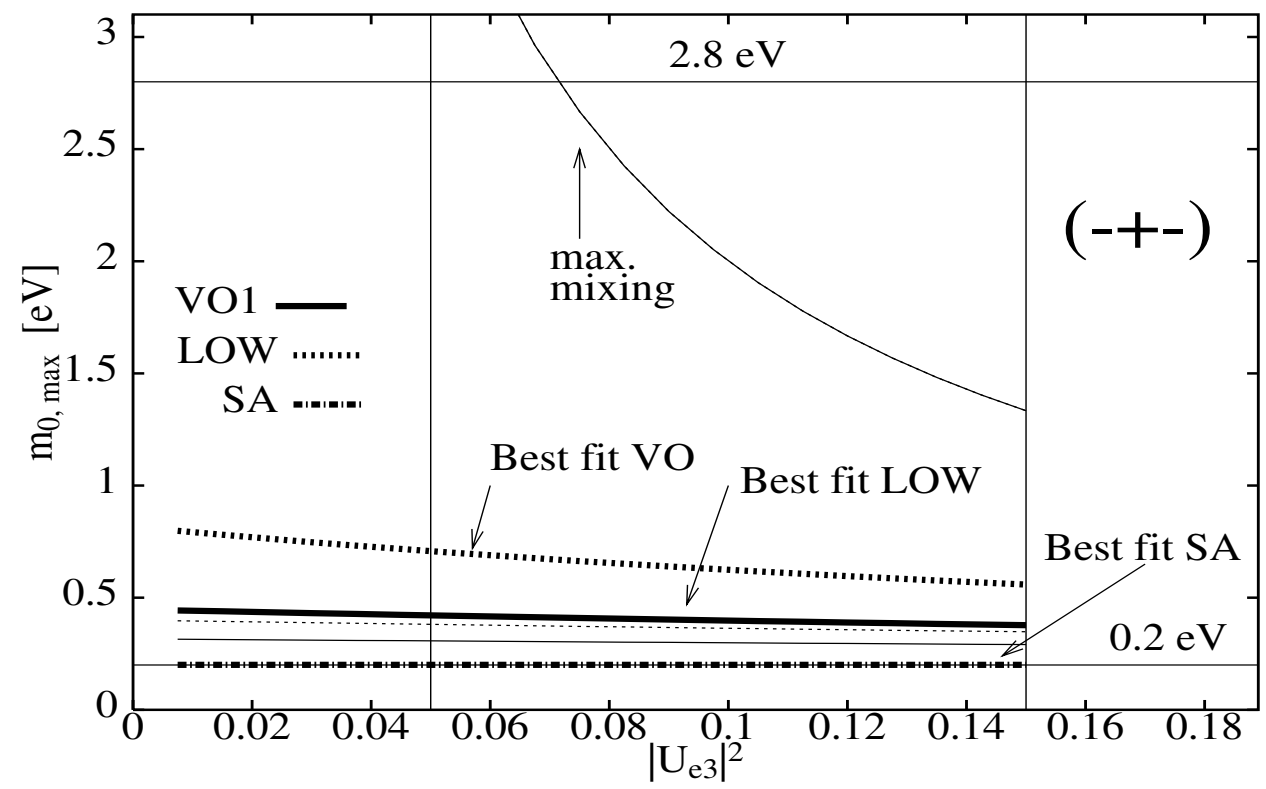

Figure 4: Same as the previous figure for the $(-+-)$ signature. Again, the LA plot is very similar to VO1 and the VO2 option is identical to VO1 option in the $(+--)$ case. 


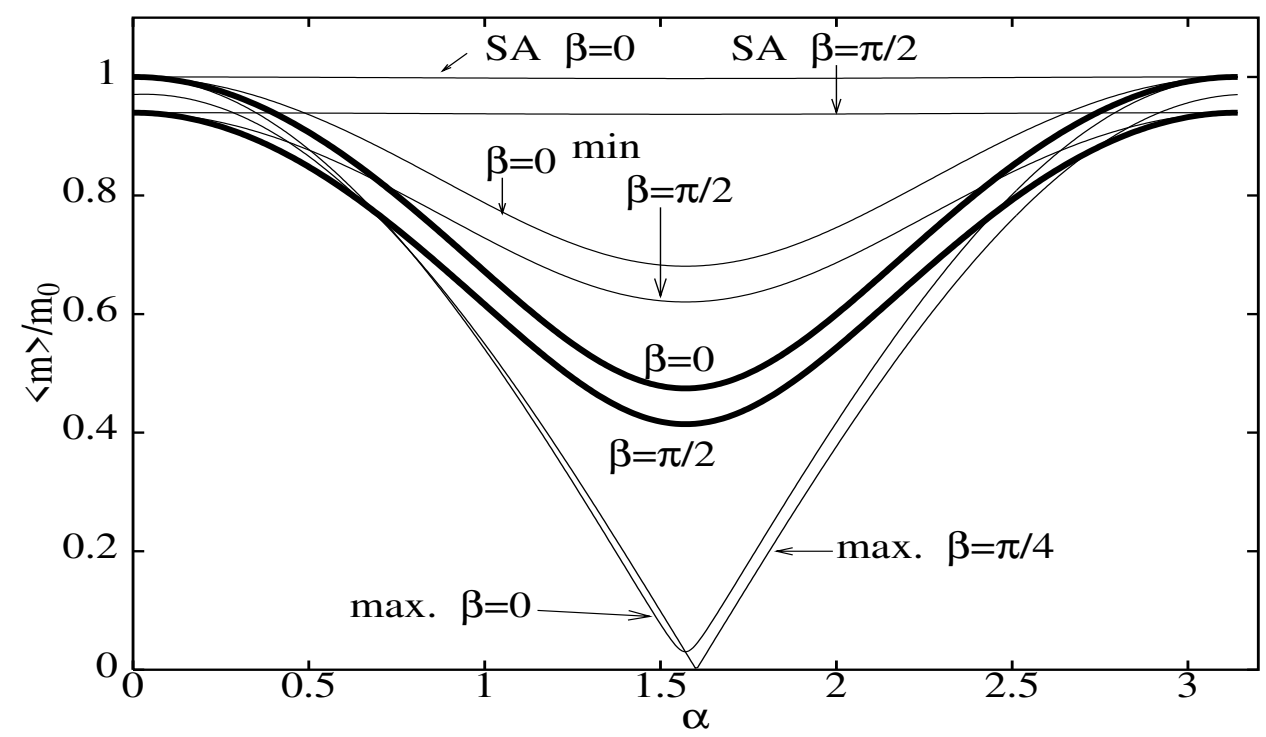

Figure 5: $\tilde{m}$ as a function of one phase $\alpha$ for different values of the second phase $\beta$. Displayed are the SA solution for $\beta=0$ and $\beta=\pi / 2$ (the two straight lines) and the LA solution (best fit point are the thick lines) for the minimal allowed $\sin ^{2} 2 \theta_{\odot}$, maximal mixing and the best fit point (thick lines). Note that maximal mixing is also allowed for VO and LOW. We assumed $\left|U_{e 3}\right|^{2}=0.03$.

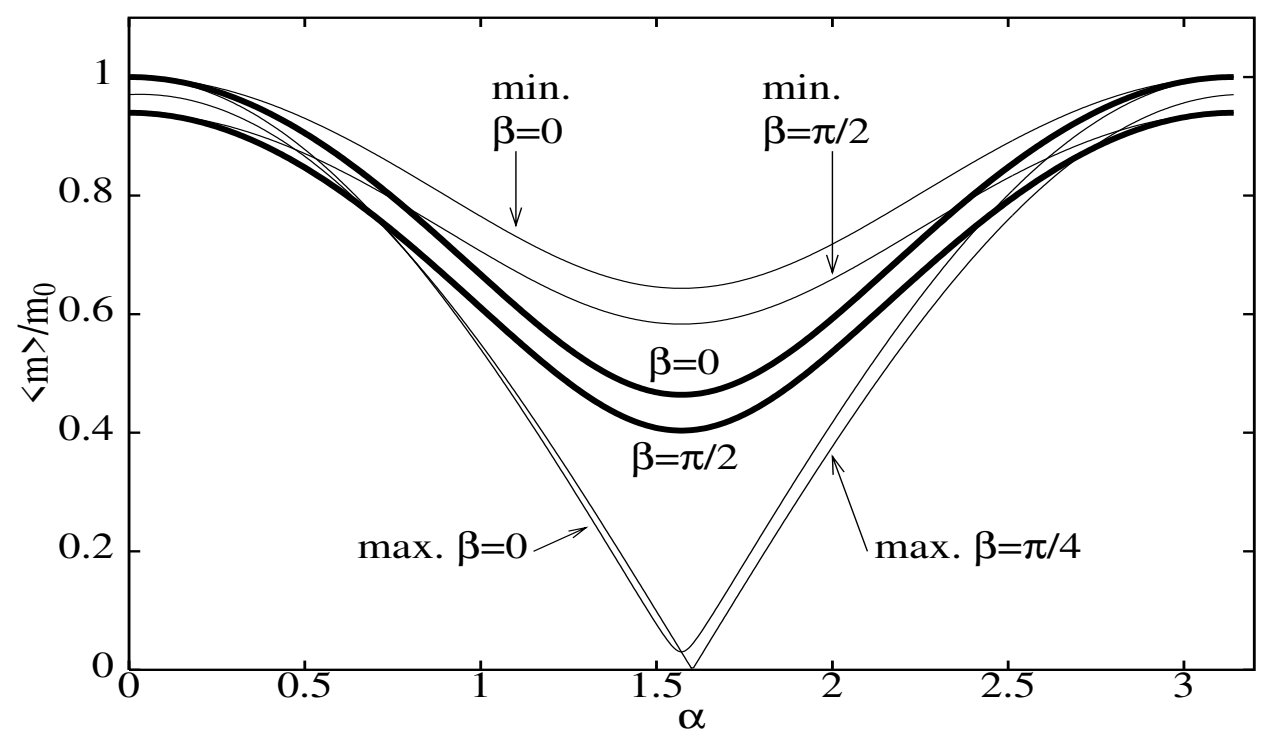

Figure 6: Same as the previous figure for the VO solution. 


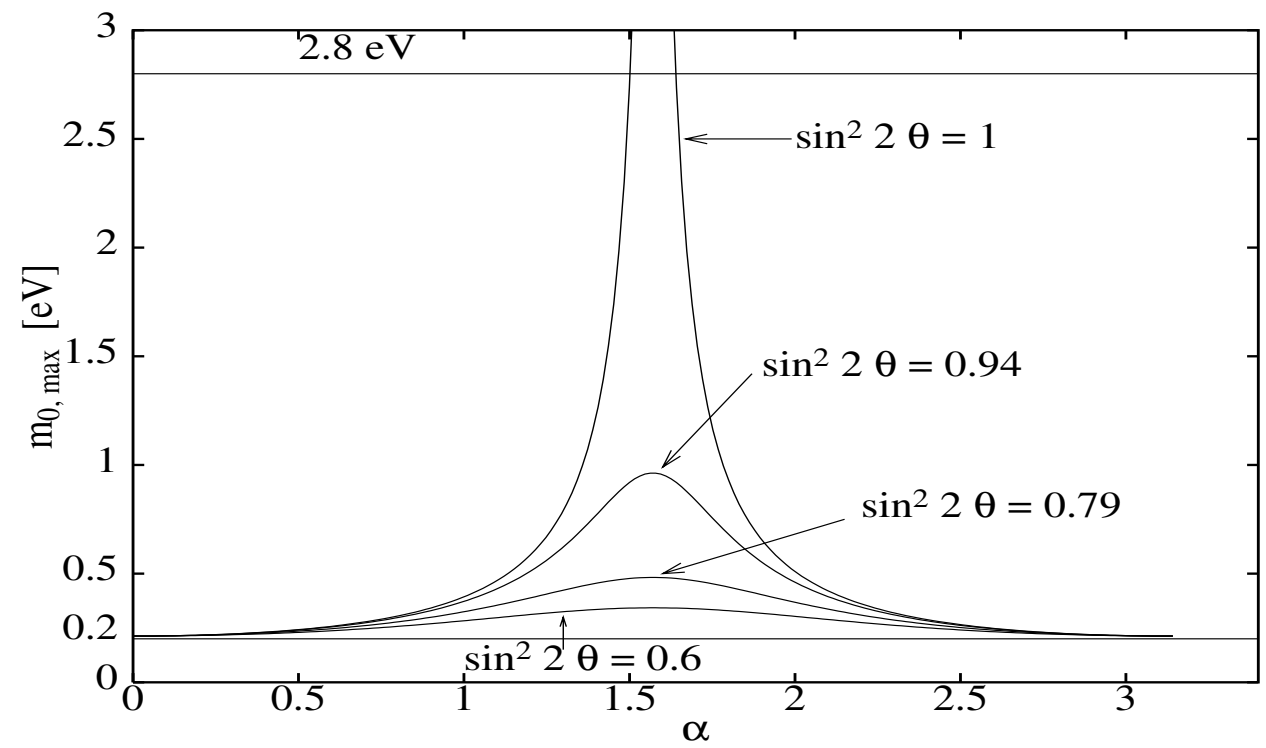

Figure 7: Maximal $m_{0}$ as a function of $\alpha$ for $\beta=\pi / 2$ and $\left|U_{e 3}\right|^{2}=0.03$ for different $\sin ^{2} 2 \theta_{\odot}$. Allowed is the area under the respective curve.

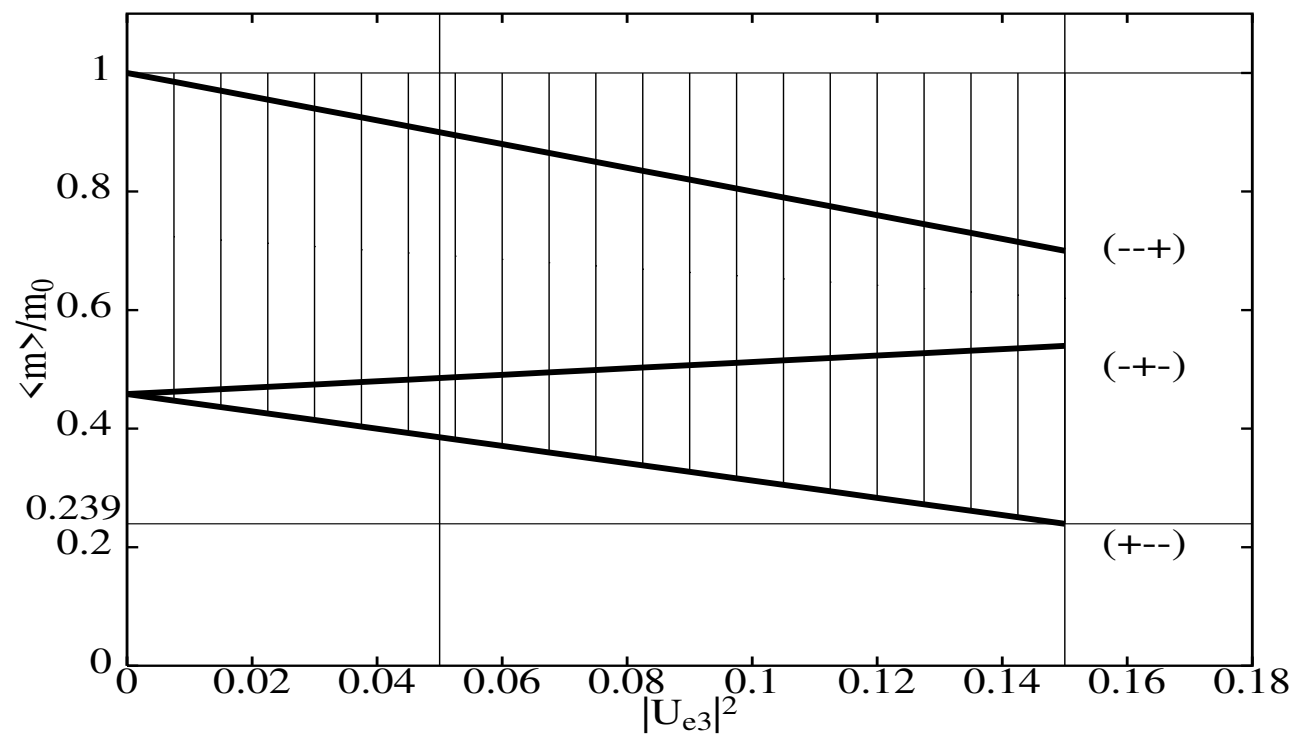

Figure 8: $\tilde{m}$ as a function of $\left|U_{e 3}\right|^{2}$ for the best-fit point of the LA solution. Displayed is the range for the $C P$-violating case and all $C P$-conserving parity configurations except the trivial $(+++)$ case. Note that the range of $\tilde{m}$ accessible by currently planned experiments is $7 \cdot 10^{-4}$ to 0.4 . 


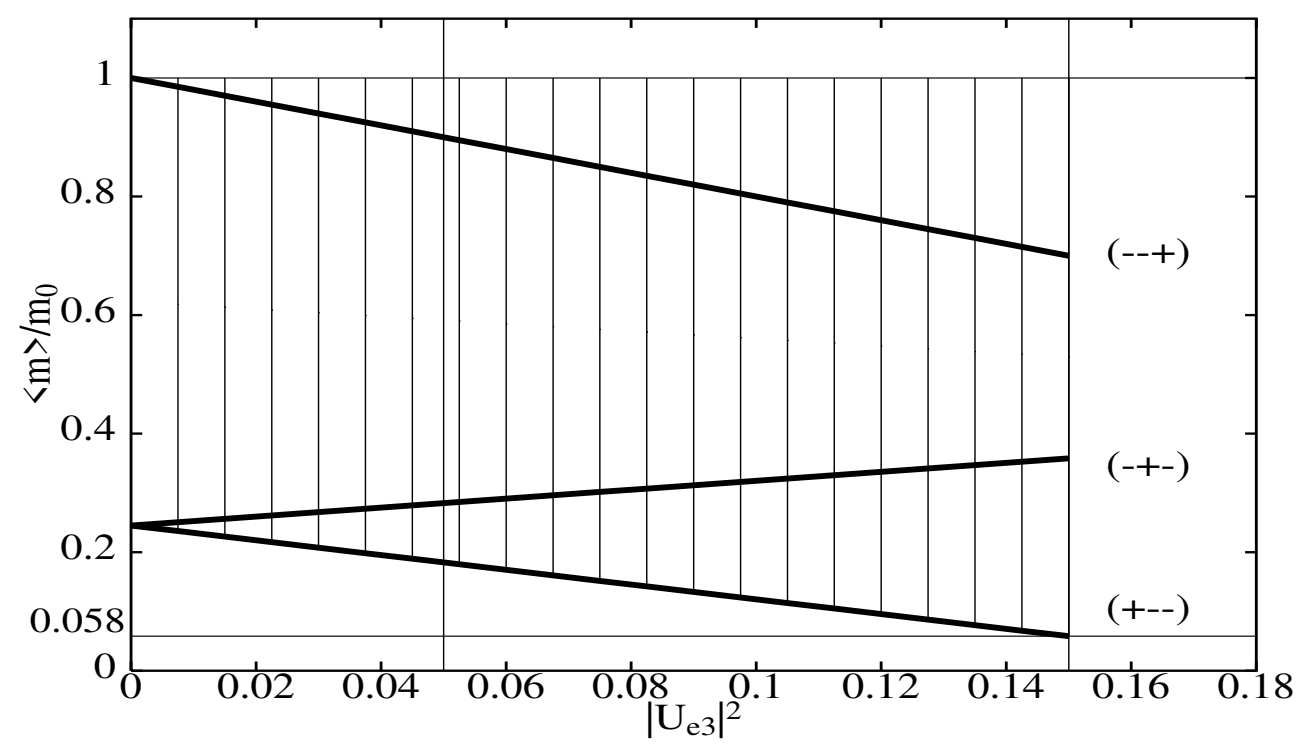

Figure 9: Same as the previous figure for the LOW solution.

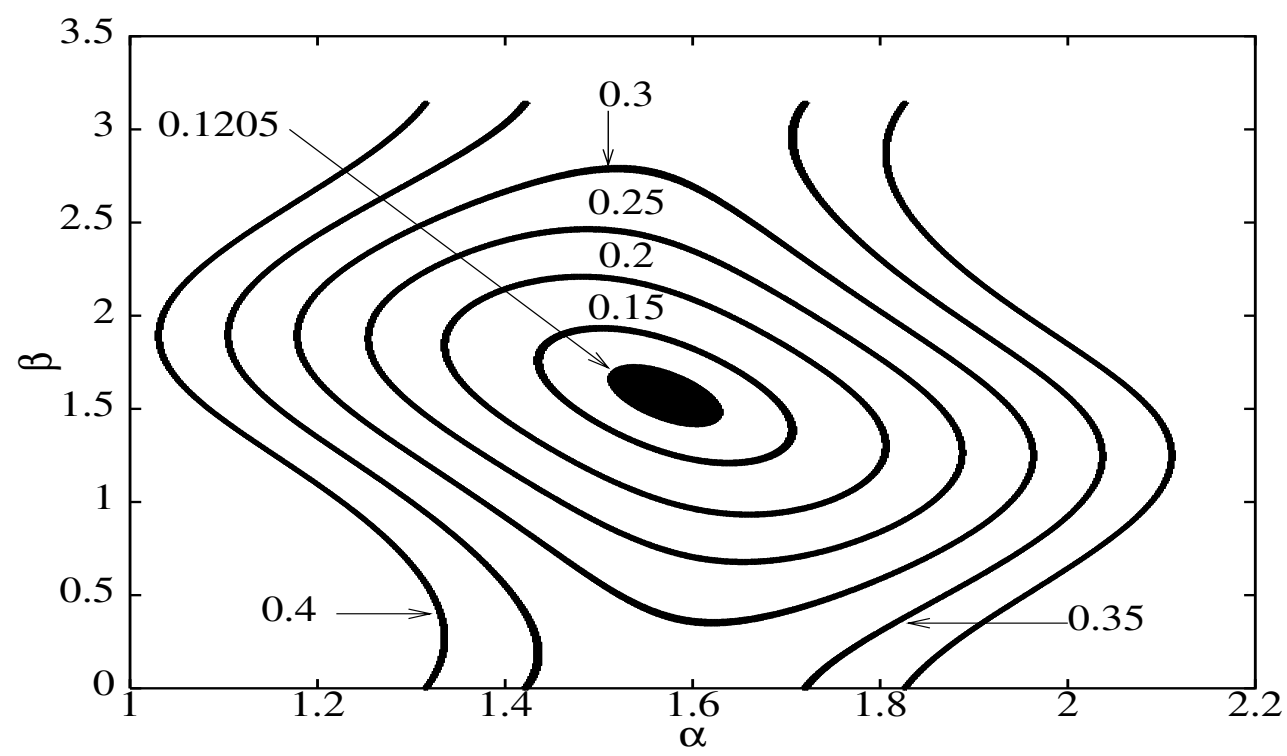

Figure 10: "Iso- $\tilde{m}$ " lines in the $\alpha-\beta$ space for the best-fit values of the LOW solution and $\left|U_{e 3}\right|^{2}=0.1$. For $\tilde{m}=0.1205$ a precision of $5 \%$ is assumed, the other values are for an exact measurement. 